\title{
Correspondances d'Eugène Green. La poésie probablement
}

Didier Coureau

\section{(2) OpenEdition}

12 Journals

Édition électronique

URL : https://journals.openedition.org/recherchestravaux/682

DOI : 10.4000/recherchestravaux.682

ISSN : 1969-6434

Éditeur

UGA Éditions/Université Grenoble Alpes

\section{Édition imprimée}

Date de publication : 1 novembre 2014

Pagination : 107-124

ISBN : 978-2-84310-282-0

ISSN : 0151-1874

Référence électronique

Didier Coureau, «Correspondances d'Eugène Green. La poésie probablement », Recherches \& Travaux [En ligne], 84 | 2014, mis en ligne le 01 avril 2016, consulté le 29 octobre 2021. URL : http://

journals.openedition.org/recherchestravaux/682; DOI : https://doi.org/10.4000/recherchestravaux 682 
Didier Coureau

Université Grenoble Alpes

\section{Correspondances d'Eugène Green La poésie probablement}

Présence veut dire lien, battement, existence; elle émerge du visible et de l'invisible. Je la reconnais dans un livre, de même que dans un tableau, une maison, un objet. Et à l'intérieur d'une ombre, de la lumière, du silence.

Silvia Baron Supervielle ${ }^{\mathrm{I}}$

\section{Le cinéma, la poésie}

Dans un entretien de 20I3, Eugène Green termine l'énumération de ses projets en affirmant : "j'aimerais aussi écrire sur la poésie car, au fond, tout ce que je fais artistiquement, y est lié2 ». Il avait déjà pu, à d'autres moments, relier sa pratique de la poésie, depuis l'adolescence, et son passage au cinéma pensé comme forme de poésie. La nécessité s’impose dès lors d'approcher la poétique de Green à travers la poésie de son cinéma, ou de son "cinématographe» puisque, après Robert Bresson et Jean Cocteau, il préfère utiliser ce terme.

Le film Le Monde vivant (2003) de Green est ouvert par la citation de l'un des grands poètes mystiques allemand, Maître Eckhart : "Si Dieu manquait à sa parole, / sa vérité, manquerait à sa divinité, / et ne serait pas Dieu, car il est sa / parole et sa vérité.»; Correspondances (2007) s'ouvre sur une citation de

I. S. Baron Supervielle, Le Pays de l'écriture, Paris, Seuil, 2002, p. 270.

2. F. Gris, "Entretien avec Eugène Green" [en ligne], Écrivains-cinéastes, n 7 de Revue critique de fixxion française contemporaine, J.-L. Jeannelle, M. C. Flinn (dir.), décembre 2013, p. I45. Disponible sur < www.revue-critique-de-fixxion-francaise-contemporaine.org/rcffcl article/view/fxo7.I4/776> (consulté le 25/06/20I4). 
Rainer Maria Rilke (dont il sera plus tard question). Le recueil de poésie de Green, lié au cinéma, Le Présent de la parole, s'ouvre quant à lui par une citation du père António Vieira : «Ce qui entre par les oreilles se crée, ce qui entre par les yeux s'impose 3 .» Vieira, poète portugais, a été pour beaucoup dans la transmission poétique du mythe de l'encoberto, du Roi caché (Dom Sebastiāo) qui doit revenir dans le monde, qu'aborde Green dans La Religieuse portugaise. Mythe lié au sentiment de la saudade, dont les fados choisis par Green dans le même film sont empreints. Dans des propos relevés dans des entretiens autour de ses films, Green cite des poètes l'ayant influencé, comme Fernando Pessoa : «Ayant appris le portugais, j'entre dans le monde de Pessoa, au point d'en faire partie4.»; ou, parmi les poètes contemporains : «[...] Bonnefoy et Novarina me touchent : chez eux le verbe incarné rejoint l'image`".

En dehors de ces citations, dans la réflexion qu'il mène dans ses essais, à côté de ses pratiques artistiques (cinéma, théâtre, lecture, conte, nouvelle, roman), Green s'appuie sur plusieurs poètes qui alimentent sa propre recherche sur le passage de la poésie textuelle à la poésie cinématographique. Maurice Maeterlinck, Paul Claudel, Victor Segalen, Stéphane Mallarmé sont à ce titre cités. À propos des deux premiers, Green écrit :

La nudité du langage de Maeterlinck constitue un style idéal pour des dialogues de cinéma, dans la mesure où la parole qui «cache» les images qu’elle porte, suggérant plus qu'elle ne dit, fournit une énergie qui appelle l'effet visible, et qui fonctionne grâce à lui. Le style de Claudel en revanche, fondé sur un foisonnement d'images communiquées par le verbe, n'est pas cinématographique, car on ne peut assiéger en même temps de fragments visibles l'oreille et l'œil. Mais, si elle ne peut fonctionner au cinéma, cette parole imagée en porte néanmoins l'idée, car elle suggère que le secret du monde peut se livrer à l'œil contemplatif'.

Du troisième, Victor Segalen, il remarque que son Essai sur le mystérieux s'approche encore davantage que son Essai sur l'exotisme "du problème de l'art cinématographique et de ce qu'il peut représenter»:

«Point de départ : le mystérieux ne réside pas tant dans un "monde essentiellement mystérieux" que dans le Moment où ce monde vient confronter le réel.» Cette "confrontation » entre "un monde mystérieux » et «le réel», ou ce qu’on prend en

3. E. Green, Le Présent de la parole, précédé de Les Lieux communs, Paris, Melville-Léo Scheer, 2004, p. 85 .

4. E. Green «Mes dates clés" [en ligne] Libération, 26/II/2003. Disponible sur <http:// next.liberation.fr/cinema/2003/II/26/mes-dates-cles-par-eugene-green_453208> (consulté le 26/06/20I4).

5. Ibid.

6. E. Green, Présences. Essai sur la nature du cinéma, Paris, Desclée de Brouwer - Cahiers du cinéma, coll. "texte et voix», 2003, p. II3-II4 
général pour tel, définit précisément la nature de la Nature dont le cinématographe peut proposer la représentation ${ }^{7}[\ldots]$

Mais c'est Stéphane Mallarmé qui occupe la place centrale de cette théorie, puisque Green va jusqu’à le considérer comme «l'inventeur virtuel de l'art du cinéma", ou comme "grand cinéaste», dans le poème "Le cinéma de Stéphane Mallarmés", ou encore en tant que "[l']un des premiers métaphysiciens du cinéma ${ }^{9}$. Green présente cette thèse dans l'étude d'Un coup de dés jamais n'abolira le hasard, qui est selon lui un échec puisque Mallarmé y «abandonne l'oralité de la poésie pour la rendre visuelle [...]», mais est aussi, "paradoxalement", une réussite car il conduit d'une certaine manière au cinématographe. Mallarmé s'est en effet

[...] rendu compte que dans le monde où il vivait le verbe captif ne pouvait plus s'incarner directement. Son salut, et aussi donc celui du poète et de l'homme, passait par l'image. Or, l'art qui permet au verbe de s'incarner dans la matière du monde et de se faire ainsi image, c'est précisément le cinématographe ${ }^{\mathrm{TO}}[\ldots]$.

La jonction entre la poésie annonçant le cinématographe, et le cinématographe lui-même, se fait au sein même de la poésie écrite par Green, à la fin d'un poème intitulé "À la mémoire de Robert Bresson", où les âmes de Mallarmé et de Bresson se rejoignent :

Le disparu, qui a créé son art,

A rendu à nos yeux le vent visible,

Comme ce cygne absurde et solitaire,

Fragile et incongru en plein hiver,

Qui fait le tour de l'île.

Ceux que le vent fait souffrir, ce sont ceux

Qui le font voir pour l'avoir entendu,

Car c'est le verbe, et il souffle où il veut ${ }^{\mathrm{II}}$.

\section{«Mini-film», poème filmique}

Correspondances, que Green désigne comme un «mini-film», dont les trenteneuf minutes l'écartent des nécessités purement narratives, pourrait s'apparenter à un poème filmique. Il est né d'une commande faite à trois cinéastes par le

7. Ibid., p. II7-II8.

8. E. Green, Le Présent de la parole, ouvr. cité, p. I20.

9. E. Green, Présences, ouvr. cité, p. IIo.

Io. Ibid., p. I05.

II. E. Green, Le Présent de la parole, ouvr. cité, p. I57. 
festival international de Jeonju (Corée du Sud), et s'est trouvé réuni, sous le titre Memories, à d'autres œuvres brèves de Pedro Costa et Harun Farocki.

Le film de Green confronte deux personnages - ou deux figures - dans un échange épistolaire, qui passe en fait par l'entremise de deux ordinateurs, claviers et écrans compris et, surtout, par l'usage de la voix off. Un jeune homme, interprété par François Rivière, Virgile (dont le prénom rappelle le nom de l'auteur des Bucoliques) correspond avec une jeune femme, Blanche, interprétée par Delphine Hecquet. Deux autres personnages les accompagnent, jouant un rôle essentiel autour de Blanche : sa mère (Christelle Prot), avec laquelle elle entretient, dans le même espace, une relation silencieuse (pas même de voix offici), et Eustache (Clément Cogitore), un autre jeune homme, présence fantomatique et muette. Un chat (Teshi, nommé au générique) enfin, celui de Blanche, occupe une véritable place de personnage, tant sa présence est intense en plusieurs moments du film.

Tout commence dans le noir, bientôt troublé par la lueur d'une bougie qui, elle-même, est ensuite transportée jusqu'à l'écran d'ordinateur. À plusieurs reprises, dans ce contraste des lumières, celle venant de la bougie, et celle de l'écran, Virgile joue le rôle du poète, se retrouvant dans la position de ce «rêveur de flamme» que décrit Gaston Bachelard dans La Flamme d'une chandelle:

Le rêveur de flamme unit ce qu'il voit et ce qu'il a vu. Il connaît la fusion de l'imagination et de la mémoire. Il s'ouvre alors à toutes les aventures de la rêverie; il accepte l'aide des grands rêveurs, il entre dans le monde des poètes. Dès lors la rêverie de la flamme, si unitaire en son principe, devient d'une foisonnante multiplicitét .

\section{Correspondance épistolaire}

Green est américain d'origine, mais il s'est fait naturaliser français, ayant reconnu cette langue comme sa langue véritable. Une autre langue, le portugais, lui importe également, son long-métrage La Religieuse portugaise en témoigne. Ce film se fonde sur les mystérieuses Lettres de la religieuse portugaise attribuées à Guilleragues (1669). Sorti en 20IO, il prolonge en quelque sorte Correspondances, dans sa réflexion sur l'échange épistolaire.

Un court-circuit se crée entre la forme classique de communication par lettres et le médium ordinateur issu du plus extrême contemporain. Green, qui s'est créé un langage très singulier, dénomme la messagerie d'Internet la «malleposte électronique». Ce jeu sur les mots, déjà en soi un travail poétique, se

I2. G. Bachelard, La Flamme d'une chandelle, Paris, PUF, coll. "Quadrige», I996, p. I2. 
trouve dans Correspondances tout d'abord au niveau de l'ordinateur où certains fragments textuels peuvent se lire dans des très gros plans. «lhommedesmers@ Courrierfroid.com », est l'adresse électronique de Virgile, et «blanche.loiseau@ Courrierchaud.com»- où se comprend aisément de quoi «courrierchaud» est la traduction - celui de Blanche. Comme à son habitude, Green aime dénoncer et transformer le moindre anglicisme ou américanisme, ce qu'il appelle «le sabir anglo-klaxon". Ce qui se retrouve encore sur la page de l'ordinateur où le site qui s'affiche est renommé «Barbare», évocation d'un grand moteur de recherche américain, Green appelant dans son langage réinventé les États-Unis d'Amérique la Barbarie (les "movies», «bougeants», «Hollywood», «Bois de Houx»). Le menu propose par ailleurs comme entrées, «recherche barbare» ou "présences électroniques», et détaille à la suite de «rechercher dans» : «toile», "pages francophones", "pages : France», pour revenir du côté de la langue française, malgré le médium employé.

Le film en son ensemble se fonde sur la correspondance écrite - le clavier de l'ordinateur a remplacé dans cet échange la plume et le stylo - et entremêle expressions contemporaines et extrême courtoisie, comme le fait d'user du vouvoiement entre une jeune fille et un jeune homme de vingt ans. Cette idée de correspondance occupe une place essentielle dans Poétique du cinématographe de Green, puisque l'exergue de l'ouvrage est une citation de Pascal sur cette thématique : "Quand on surprend une lettre importante où l'on trouve un sens clair, et où il est dit néanmoins que le sens en est voilé et obscurci, qu'il est caché en sorte qu'on verra cette lettre sans la voir, et qu'on l'entendra sans l'entendre, que doit-on penser sinon que c'est un chiffre à double sens ${ }^{13}$ ? » La correspondance, dont il est question dans le film, se présente cependant de manière étrange. Si certains messages sont amorcés par écrit, et que les mains en très gros plans sont souvent vues sur le clavier, c'est par la parole que les échanges sont perçus. Les voix off communiquent, sans que jamais les personnages ne soient vus en train de parler, pas même ceux qui se trouvent dans un même espace : Blanche et sa mère, Blanche et Eustache, Eustache et Virgile. L'échange prend une teneur presque médiumnique ou télépathique, qui outrepasse la simple relation par courriers électroniques. Certains messages se font d'ailleurs attendre, parfois par la jeune fille, parfois par le jeune homme, comme de véritables envois postaux. Mais la voix introduit une autre dimension poétique, qui pourrait rejoindre la réflexion d'André Rolland de Renéville selon laquelle : «La parole peut se définir comme la réfraction de l'idée passant du monde intellectuel dans celui de la

13. E. Green, Poétique du cinématographe, Arles, Actes Sud, 2009, p. 9. 
matière ${ }^{14}$.» Propos qu'il démontre à partir d'une analyse de Mallarmé, en assemblant différemment quelques-uns de ses vers («Hérodiade»), «Une voix / Encore dans les plis jaunes de la pensée / S'élève. ", afin d'étayer sa démonstration :

Nos remarques sur la constitution de la parole, qui ne devient telle qu'au moment de son apparition dans le monde physique, nous permettent de pénétrer le sens parfait de ces vers. La voix que le poète entend venir du fond de son inconscient ne se formule pas encore en paroles; elle participe à l'absolu de la pensée et s'apprête à descendre dans le monde formel où sa transformation d'idée en parole doit s'accompliris.

La voix off, dans Correspondances, peut être perçue comme un état intermédiaire entre voix intérieure et formulation par la parole, au-delà de l'échange épistolaire-électronique, dans la rencontre entre les deux jeunes gens qui sera rendue possible au sein du monde réel.

Dans ce tremblement de la chandelle, et de ce tremblement de l'écran de l'ordinateur, naît le verbe dans les vibrations de la voix. Blanche pourrait ressembler à cette femme apparition, nommée "La Blanche-par-amour" par le poète Joë Bousquet qui écrit : «Le souvenir de sa voix est autour de moi comme un bruit de source et peut-être le rythme en restera attaché au silence, car je le perçois comme l'écho de quelque chose d'intérieur ${ }^{16}$."

\section{Correspondances baudelairiennes}

Si de correspondance il est question dans le film, constituant ainsi ce que l'on pourrait nommer un poème épistolaire, les correspondances au pluriel du titre évoquent aussi celles pensées par Charles Baudelaire, clefs de sa poésie, de sa poétique et de son esthétique :

Comme de longs échos qui de loin se confondent

Dans une ténébreuse et profonde unité,

Vaste comme la nuit et comme la clarté,

Les parfums, les couleurs et les sons se répondent ${ }^{17}$.

Ces correspondances baudelairiennes donnent également des clefs pour appréhender le cinéma de Green, et les accords multiples qui se créent au sein des plans de son film et, surtout, dans la relation entre les plans, constituant

I4. A. R. de Renéville, L'Expérience poétique (ou le feu secret du langage), Saint-Aubin, Le Grand Souffle, coll. «Littérature», 2004, p. 30.

I5. Ibid., p. 32.

I6. J. Bousquet, Le Meneur de lune, Paris, Albin Michel, coll. «Esthétique», 1989, p. 59.

17. Ch. Baudelaire, "Correspondances", dans Les Fleurs du Mal. 
alors une forme de poésie filmique. Accords entre sons et images, sons et sons, images et images, cinéma et autres arts.

Correspondances est bien de l'ordre de la poésie amoureuse. Ce qui est mis en jeu c'est la naissance d'un amour issu d'une relation épistolaire dans laquelle les mots, peu à peu, permettent de dénouer les liens virtuels qui tenaient attachée Blanche. Cette parole révélatrice apparaît dans une diction qui évite la psychologisation, Green s'inscrivant en cela, à sa manière, dans la lignée de Bresson. Comme lui, il recherche une mécanique de la diction, mais ajoute un petit artifice qui lui est propre, celui de faire prononcer toutes les liaisons entre les mots, même les plus inusitées. Par exemple, faire la liaison entre le «n» final du mot maman et le mot suivant dans la phrase : «maman apprécie» est alors entendu "maman n'apprécie». Artifice qui a aussi une incidence sur la rythmique de la phrase.

\section{Composition poétique-filmique}

De la relation du cinéma à la poésie, Green écrit encore :

La poésie, c'est l'apparition du verbe qui se cache dans les mots. Le cinématographe, c'est la parole faite image. Ainsi, la poésie contenait virtuellement l'art dont on attribue l'invention aux frères Lumière, tandis que celui-ci est une forme contemporaine de poésie. Mais les moyens utilisés pour aboutir au même résultat sont tellement différents, que toute comparaison devient stérile ${ }^{18}$.

Ce passage, très riche, réaffirme une proximité réelle entre cinéma et poésie, tout en spécifiant qu'une comparaison terme à terme serait impossible. Si cela est exact, dans le sens ou le mot n'est pas l'image, il existe cependant certaines conceptions du plan, et de certains liens qui se font entre les plans, qui peuvent faire écho à la composition écrite du poème. Green se contredit d'ailleurs quelque peu quand, dans le même ouvrage, il souligne :

Le plan est l'équivalent cinématographique du vers, et doit toujours être entouré d'un vide, semblable au silence qui précède et qui suit chaque unité d'un poème. Comme des plans se succèdent sur l'écran sans aucune absence d'image, ils doivent contenir le vide en eux-mêmes. Ils doivent comporter aussi clairement lisible, leur structure rythmique ${ }^{19}$.

Il rejoint ainsi plusieurs cinéastes, tel Bresson qui pense que "[...] les images ne peuvent pas être poétiques car la poésie vient des rapports, des relations, pas d'une image. De même qu'en littérature, la poésie ne vient pas d'un mot,

18. E. Green, Poétique du cinématographe, ouvr. cité, p. 4I.

I9. Ibid., p. 97. 
mais des rapports entre les mots ${ }^{20}$ ", ou Jean-Daniel Pollet qui affirme que "[...] l'image mot conduit naturellement à construire des images phrases à peu près libérées des films essentiellement narratiff ${ }^{2 \mathrm{I}} »$.

\section{Correspondances : objets et matières}

Une véritable composition poétique préside au montage filmique. Ainsi en est-il de certains plans d'objets, qui reviennent à intervalles réguliers, scandant le rythme du film, en créant de véritables rimes ou consonances visuelles. Dans Le jour se lève de Marcel Carné, André Bazin, après avoir attaché une importance toute particulière aux objets en présence, dans la chambre où le personnage est enfermé (il demandait aux spectateurs, dans le cadre de son ciné-club, de se remémorer ces objets), écrit :

On voit comment le réalisme de Carné sait, tout en restant minutieusement fidèle à la vraisemblance de son décor, le transposer poétiquement, non pas en le modifiant par une transposition formelle et picturale [...], mais en dégageant sa poésie immanente, en le contraignant à révéler de secrets accords avec le drame ${ }^{22}$.

Bazin concluait que "c'est en ce sens qu'on peut parler de "réalisme poétique" $[. .$.$] ", et précisait que "plutôt que de symbolisme c'est d'une métaphy-$ sique du décor qu'il faudrait parler", ce qui s’accorde au cinéma de Green dans Correspondances, où tous les objets ont une fonction poétique manifeste.

Parmi ceux-ci, deux bonnets de laine occupent une place cruciale. Un bonnet rouge posé sur le marbre gris foncé d'une tablette de cheminée, un bonnet bleu posé sur le bois d'une table. Le bonnet rouge est celui de Virgile, le bonnet bleu est celui d'Eustache, son rival virtuel puisque disparu, mais réapparaissant sous forme de fantôme. À quatre reprises les bonnets apparaissent dans un enchaînement de deux plans les montrant, l'un dans l'appartement de Blanche, l'autre dans l'appartement de Virgile : bonnet rouge / bonnet bleu, bonnet bleu / bonnet rouge, bonnet rouge / bonnet bleu, bonnet rouge / bonnet bleu. Puis, séparés par plusieurs plans : bonnet bleu et bonnet rouge. Il est à noter qu'entre le bleu et le rouge figure Blanche, comme une affirmation par le jeu des couleurs de l'attachement de Green au bleu blanc rouge du drapeau français!

20. R. Bresson, «Une mise en scène n’est pas un art», intervention à l'IDHEC en décembre 1955, Hommage à Robert Bresson, supplément aux Cahiers du cinéma, $\mathrm{n}^{\circ}$ 543, février 2000, p. 6.

2I. J.-D. Pollet, «Le complexe de Robinson", Cahiers du cinéma, n 509 , janvier I997, p. 35 .

22. A. Bazin, «Le décor est un acteur» [en ligne], paru dans Ciné-Club, $\mathrm{n}^{\circ} \mathrm{I}$, décembre I949, p. 2. Disponible sur <http://www.marcel-carne.com/presse/bazin49.html> (consulté le 26/06/20I4). 
La couleur elle-même participe du travail de révélation constamment mis en ouvre par Green, qui note : «Dans toute utilisation cinématographique de la couleur, on devrait apercevoir non pas la qualité matérielle de la surface des choses, mais un élément intérieur que l'art a rendu visible ${ }^{23}$."

Il serait possible d'établir la liste précise des occurrences se créant dans la répétition de la vision de différents objets, qui s'inscrivent au cœur de ce poème codé qui s'écrit aussi "EN IMAGES EN MOUVEMENT ET EN SONS", selon la description de l'écriture d'un film que donne, en lettres majuscules, Bresson ${ }^{24}$, dont une autre note indique : «Tous ces effets que tu peux tirer de la répétition (d'une image, d'un son ${ }^{25}$ )». Il faut préciser quels sont ces objets, ces choses - outre les bonnets - qui permettent d'entretisser les deux espaces disjoints du film : la bougie (Virgile) et l'écran de l'ordinateur (Virgile, Blanche); les chaussures, filmées dans un cadrage au ras du sol, dont un précédent se trouve dans le film de Manoel de Oliveira Je rentre à la maison (Virgile et Blanche); l'oreiller et les draps bleus (Virgile), le dessus-de-lit et les draps blancs (Blanche); les écouteurs d'un baladeur (Blanche), une chaîne stéréophonique (Virgile); une écharpe noire (Virgile), un châle bleu (Blanche); un fauteuil et une chaise (Blanche), une chaise et une chaise de bureau avec cinq branches munies de roues (Virgile); un vase et une rose rouge (Blanche); des fragments de l'espace : le bas d'une porte et une prise, la poignée de cette porte, un miroir sur une cheminée, des rideaux qui masquent une fenêtre (Blanche). Dans cette image épurée, où chaque chose prend une importance considérable, le projet de Green est alors proche de celui de Pollet qui déclare que pour Méditerranée "[son] seul principe [...] était de ne filmer qu'une chose par plan. Trouver des images signes, des images mots ${ }^{26}{ }$. Chaque objet se charge d'une intensité qui s'accorde à cette intuition première de Jean Epstein, cinéaste et penseur mystique du cinéma, proche en de nombreux points de Green, lorsqu'il écrivait que "[l'] une des plus grandes puissances au cinéma est son animisme. À l'écran, il n'y a pas de nature morte. Les objets ont des attitudes ${ }^{27}$ ».

À travers les choses, les sensations de matières sont intensément perçues. Le bois du parquet, parfois lié au dessus-de-lit blanc de Blanche, ou à deux taches de lumière qui se projettent sur lui : «La lumière est une énergie qui nous permet d'appréhender visuellement la matière du monde. Dans le cinématographe, elle

23. E. Green, Poétique du cinématographe, ouvr. cité, p. 95.

24. R. Bresson, Hommage à Robert Bresson, ouvr. cité, p. I2.

25. Ibid., p. 57.

26. J.-D. Pollet, «Le complexe de Robinson», art. cité.

27. J. Epstein, «Le cinématographe vu de l'Etna», dans Écrits sur le cinéma, I, Paris, Seghers, coll. "Cinémaclub», I974, p. I34. 
rend saisissable aussi l'énergie que la matière dégage ${ }^{28}$ ", écrit Green; le marbre de la tablette de la cheminée où se trouve le bonnet de Virgile, et le bois de la table où se trouve le bonnet d'Eustache; la laine des bonnets; la texture des draps, des rideaux; le bois des autres meubles... Des motifs se dessinent et se répètent : la spirale du bras du fauteuil de Blanche, et les coquillages et spirales, finement tissés sur le dessus-de-lit de la jeune femme, qui viennent s'accorder aux effets de plis des draps et rideaux. Ici se révèle déjà la pensée par Green de l'«oxymore baroque» dont il précise : "C'est à mon sens la clé de cette civilisation, ce qui m'intéresse le plus, et qui est le plus important pour nous aujourd'hui : la conscience de l'existence d'une réalité spirituelle dans la matière ${ }^{29}$.»

\section{Correspondances : corps et âmes}

"Un seul mystère des personnes et des objets ${ }^{30}$ ", notait Bresson. Qu'en est-il de la correspondance entre les corps? Comme chez Bresson les personnages de Green sont captés dans une certaine immobilité et une rigidité, trahies parfois par quelques gestes très précisément observés, dont certains laissent transparaître une énergie nerveuse telle qu'Epstein la revendiquait dans les gros plans et très gros plans en mouvement : poing de Virgile qui frappe la paume de son autre main, doigts de Virgile qui brisent des allumettes. Mais, comme chez Bresson également, cette maîtrise de la parole et du corps laisse transparaître une réelle profondeur, où les yeux et les regards, ainsi que les mains, jouent un rôle clef. Un choix récurrent est celui de la frontalité du cadrage, dans la création d'un champ-contrechamp qui réunit les personnages séparés dans l'espace respectif de leurs appartements, mais qui inclut aussi celui qui regarde le film, en n'évitant pas les regards-caméras. "Quand un personnage affronte son intériorité», écrit Green, «il est logique qu’il regarde la caméra - l'œil d'un spectateur - car le propre du cinématographe est de rendre visible ce qui dans le monde reste cachési ${ }^{11}$. Bresson écrivait, avec citation des Essais de Montaigne à l'appui (Green rapproche d'ailleurs sa poésie des textes de l'inventeur de l'essai) : "Modèle. "Tout face ${ }^{32}$ "."

À la manière des bonnets et d'autres objets qui permettent d'établir passages et jeux de correspondances entre lieux séparés, la composition poétique - montage

28. Ibid., p. 79.

29. "Par-delà la mort, deux amants unis par la voix», entretien avec E. Green, par J. Mandelbaum et I. Régnier, Le Monde, mercredi io novembre 2004, p. 27.

30. R. Bresson, Notes sur le cinématographe, Paris, Gallimard, I975, p. 23.

31. E. Green, Poétique du cinématographe, ouvr. cité, p. 9I.

32. R. Bresson, Hommage à Robert Bresson, ouvr. cité, p. 39. 
filmique permet de relier les êtres malgré leur séparation. Les mains de Virgile qui écrivent entrent en communication avec les mains de Blanche qui écrivent, même le clavier d'ordinateur devient ainsi véhicule d'une sensualité du toucher. Un enchaînement de plans confirme le rapprochement virtuel des deux jeunes gens quand, sur deux surfaces séparées, seuls sont visibles les avant-bras de Virgile et ceux de Blanche, et leurs mains qui semblent se diriger l'une vers l'autre. Plans abstraits qui rappellent certains plans d'Une femme mariée de Jean-Luc Godard, par la fragmentation et la sensation, tout en évoquant par cette scène amoureuse télépathique suggérée, une très belle séquence de L’Atalante de Jean Vigo, qui réunit par le montage et les postures les amants séparés. La caresse, dans Correspondances, aboutit d'un côté à resserrer les doigts sur les draps, de l'autre à la fourrure du chat, ici profondément baudelairien : «Leurs reins féconds sont plein d'étincelles magiques, / Et des parcelles d'or, ainsi qu'un sable fin, / Étoilent vaguement leurs prunelles mystiques ${ }^{33}$.» D'autres plans montrent le chat couché sur le lit ou le fauteuil, ouvrant et fermant ses yeux verts, et d'autres réminiscences baudelairiennes se font jour : «Je vois avec étonnement / Le feu de ses prunelles pâles, / Clairs fanaux, vivantes opales / Qui me contemplent fixement ${ }^{34}$.»

Un autre moment du film scelle cet amour encore virtuel, lorsque la caméra parcourt le corps de Blanche dénudé, vu en amorce de dos sur le côté gauche du champ, et de face, légèrement flou, dans un miroir où le mouvement d'appareil descendant vient bientôt laisser apparaître son buste. Un autre plan similaire montre le dos de Virgile. Deux corps nus face à leurs miroirs, mais aussi en miroir l'un de l'autre. Le miroir, objet baroque par excellence, est également le révélateur de l' «Image-Temps» selon Gilles Deleuze, point de passage entre l'actuel et le virtuel. Ce rapprochement entre les corps prolonge les très nombreux échanges suggérés, dont ceux des regards, dans les champscontrechamps, souvent effectués entre gros plans des visages. Et ce n'est certes pas un hasard si Green a choisi une comédienne aux grands yeux bleus. "Ce que le cinéma permet de plus beau, c'est de capter la lumière d'un être ${ }^{35}$ ", précise Green, comme Bresson notait : «Modèle. L'étincelle attrapée dans sa prunelle donne signification à toute sa personne ${ }^{36}$.»

Quelque chose se joue entre le visible et l'audible, qui est encore du visible, puisque pour Green, «Le cinéma, c'est la parole faite image ${ }^{37}$ », pensée partagée

33. Ch. Baudelaire, Les Fleurs du Mal, ouvr. cité.

34. Ibid., p. 55 .

35. E. Green, La Religieuse portugaise, La Riche, Diabase, coll. «Liens et résonance», 20Io, p. 156.

36. R. Bresson, Hommage à Robert Bresson, ouvr. cité, p. 94.

37. E. Green, Poétique du cinématographe, ouvr. cité, p. I4. 
par Oliveira pour qui «le son est de l'image» et "la parole, c'est de l'image (38 $^{38}$, et Pollet qui souligne : "L'Image qui parle : c'est une sensation que j'éprouve souvent, l'impression d'une parole derrière l'image ${ }^{39}$." Quand Virgile déclare à Blanche «[j']ai aperçu aussi ce qu'on voit par les oreilles", il s'en explique en précisant que cela se produit " [q] uand on devient une correspondance», c'està-dire "quand on sent qu'on devient le lien entre deux morceaux du monde", en faisant "résonner quelque chose de visible comme une parole». La correspondance se situe dans cet entre-deux de la présence et de l'absence. Dans son poème "Prison", qui semble par ailleurs renvoyer au Cantique des cantiques ( $\hat{O}$ toi ma bien-aimée, la plus belle des femmes ${ }^{40} . »$ ), Green donne à lire ces vers : "Ô toi ma bien aimée qui étais une absence, / je te retrouve ici réelle comme $\mathrm{moi}^{4^{4}}$ ! " qui laissent entendre en écho ceux de Maurice Scève : «En toi je vis, où que tu sois absente : / En moi je meurs, où que soye présent. / Tant loin sois-tu, toujours tu es présente : / Pour près que soye, encore suis-je absent ${ }^{42}$.» Dans l'entre-deux se cache et se révèle la "présence réelle», notion fondamentale pour Eugène Green, qui la construit en se fondant sur sa dimension religieuse : le corps du Christ perçu comme présent par les catholiques lors du rituel de l'Eucharistie, et dont il précise : «on saisit la présence réelle par la reconnaissance de signes. Ce qui constitue une religio, ce sont les liens permettant aux membres d'une même communauté humaine de reconnaître dans les mêmes signes une pensée spirituelle ${ }^{43}$ ». Cette idée même d'eucharistie peut rejoindre celle de la présence réelle d'un amour révélée à travers la croyance en un signe. Blanche fait en ce sens songer au personnage Blanchefleur, dont le visage est perçu par Perceval dans trois gouttes de sang - d'une oie blessée par un faucon - tombées sur la neige. Dans un livre intitulé Cinématographies, Jean-Louis Schefer donne une très belle analyse de la scène décrite par Chrétien de Troyes ${ }^{44}$ :

L'image que contemple Perceval ouvre sa mémoire [...], elle fond au soleil. Sa symbolique lointaine (ou plutôt prochaine dans le Conte du Graal) est eucharistique; cette neige et ce sang y sont l'hostie représentant la présence réelle [...].

38. "La vie est une énigme», entretien avec M. de Oliveira, par J.-M. Lalanne et Ch. Tesson, Cahiers du cinéma, $\mathrm{n}^{\circ}$ 57I, septembre 2002, p. 57.

39. J.-D. Pollet, G. Leblanc, L'EntreVues, Montreuil, L'CEil, I998, p. 48.

40. E. Green, Le Présent de la parole, ouvr. cité, p. 158 et 162.

4I. Ibid., p. I6I.

42. M. Scève, dans P. Seghers, Le Livre d'or de la poésie française (des origines à 1940), Paris, Marabout, coll. «Université», 1972, p. 80.

43. E. Green, Présences, ouvr. cité, p. 233.

44. Il faut souligner que Green, dans Le Monde vivant (2003), s'inspire de Chrétien de Troyes (on y trouve un étrange "Chevalier au lion" dont le lion est un chien, et une demoiselle enfermée dans une chapelle). Il est difficile également de ne pas penser aux films de Robert Bresson, Lancelot du lac (1974) et d'Éric Rohmer, Perceval le Gallois (1978). 
Blanchefleur sera l'hostie : l'image instable sur la neige est exactement le lieu d'une transsubstantiation ${ }^{45}$.

\section{Correspondances entre les arts}

La relation qui se noue entre Blanche et Virgile est redoublée par une autre correspondance créée entre deux icônes. Chez Virgile, qui affirme sa passion pour le cinéma, se trouve une photo extraite du film d'Olivier Assayas Irma Vep (I996), elle montre Maggie Cheung, qui interprète Irma Vep (anagramme de Vampire), personnage resurgi dans le cinéma contemporain, après avoir hanté une série de films (1915-1916) de Louis Feuillade. Originaire de Hong Kong, la comédienne rapproche le goût de Green pour le cinéma extrême-oriental, du côté de Yasujiro Ozu dont il dit se sentir extrêmement proche ( «formellement je ressemble sans doute beaucoup plus à Ozu qu’à Bresson ${ }^{46}{ }^{\circ}$ déclare-t-il), mais aussi d'autres cinématographies asiatiques comme celles de Hou Hsiao-hsien, Wong Kar Wai, Edward Yang ou Kore-Eda, et son goût pour la Nouvelle Vague française représentée ici par Jean-Pierre Léaud, réalisateur du film dans le film. Mais est aussi présente, en filigrane, l'actrice de Feuillade qui fascina les poètes, dont Robert Desnos écrivait :

Musidora, que vous étiez belle dans Les Vampires! Savez-vous que nous rêvions de vous et que, le soir venu, dans votre maillot noir, vous entriez sans frapper dans notre chambre, et qu'au réveil, le lendemain, nous cherchions la trace de la troublante souris d'hôtel qui nous avait visités ${ }^{47}$ ?

Mais Léaud évoque aussi dans son rôle de cinéaste, lourd de fatigue existentielle, un autre cinéaste qui fit appel à lui à deux reprises, pour Le Père Noël a les yeux bleus (1966) puis La Maman et la Putain (1973) : Jean Eustache. Sur le générique final du film, il est noté que la photographie de plateau de Maggie Cheung a été prise par Isabelle Weingarten, qui fut «modèle» chez Bresson dans Quatre nuits d'un rêveur (1972), puis joua dans La Maman et la Putain où Alexandre/ Léaud disait : "Une femme me plaît par exemple, parce qu'elle a joué dans un film de Bresson.» Eustache, nom du cinéaste est aussi, dans Correspondances, le prénom du fantôme qui obsède Blanche, et apparait à l'écran lorsqu'il est question pour lui de cesser de hanter les lieux de sa virtuelle présence. Le jeune

45. J.-L. Schefer, "Ce que Perceval regarde», dans Cinématographies, objets périphériques et mouvements inverses, Paris, POL, I998.

46. "Un "martien patriarche", la genèse du Monde vivant" [en ligne], entretien avec E. Green, par Ph. Chapuis, décembre 2003. Disponible sur <http://www.objectif-cinema. com/interviews/279d.php> (consulté le 25/06/20I4).

47. R. Desnos, Les Rayons et les ombres. Cinéma, Paris, Gallimard, I992, p. 84. 
homme, qui a déclaré son amour à Blanche, sans qu'elle ne l'ait jamais vu, s'est en effet suicidé (comme le personnage du Diable probablement de Bresson). Le suicide d'Eustache, le personnage de Green, renvoie dès lors au suicide d'Eustache, le cinéaste. Dans La Maman et la Putain, Alexandre répliquait : «Écoute je n'arrive pas à ne pas prendre le suicide au sérieux, pas plus que la mort, ou pas moins. Comment faut-il dire? Alors parle-moi de suicide tant que tu veux mais si j'en ris c'est que j'en ai très peur.» La conception du fantôme de Green passe par The Dead de James Joyce, qui montre «le lien qu'un être mort crée entre deux êtres vivants $4^{8}$ ", et par Les Contes de la lune vague après la pluie (1953) de Mizoguchi, où la femme morte d'un soldat revenu de la guerre apparaît, "comme une réalité naturelle» capable de transmettre une "énergie vitale ${ }^{49}$ ", avant même d'être présentée en tant que fantôme.

Au mur de la chambre de Blanche est accrochée une reproduction de La Vierge de l'Annonciation (ou L'Annonciation) d'Antonello da Messina (I476), qui vient dialoguer avec l'image d'Irma Vep / Maggie Cheung. La Vierge, dans ce tableau, comme cela est extrêmement rare dans les Annonciations, est vue seule. Sa tête et ses épaules sont recouvertes par un voile bleu, de sa main gauche elle en tient les pans fermés sur sa poitrine, de sa main droite elle esquisse un geste de la main, en signe de réception de la parole invisible. La présence de l'archange Gabriel est cependant suggérée par le soulèvement des feuilles du livre qu'elle tient ouvert devant elle sur un petit pupitre. L'exergue de Correspondances n'est-il pas la sentence de Rilke : "Einjeder Engel ist schrecklich» («Tout ange est effrayant», dans la traduction donnée par le film, dont est connue aussi la traduction "Tout ange est terrible»). Le tableau de l'Annonciation, de la parole annonciatrice, fait face à la photo d'Irma Vep, et une même mystique imprègne ces figures de la vie et de la mort. Les pages qui se tournent sous le souffle de l'ange, voilà qui relie encore la pensée occidentale et la pensée extrême-orientale. "Le vent souffle où il veut» était l'exergue du film de Bresson, Un condamné à mort s'est échappé, phrase extraite de l'évangile de Jean : «Le vent souffle où il veut : tu entends le bruit qu'il fait, mais tu ne sens pas d'où il vient ni où il va. Il en est ainsi de tout homme qui est né du souffle de l'esprit ${ }^{5 \circ}$.» Mais Bresson écrivait aussi dans ses notes : "TRADUIRE le vent invisible par l'eau qu'il sculpte en passant ${ }^{5 I}$.» Idée que l'on peut déjà trouver chez Confucius : "Quand le vent passe, les herbes s'inclinent ${ }^{52}$.» $\mathrm{Ou}$,

48. E. Green, Présences, ouvr. cité, p. 25.

49. Ibid., p. I9-20.

50. Jean, chapitre 3.8, La Bible, Nouveau Testament.

5I. R. Bresson, Hommage à Robert Bresson, ouvr. cité, p. 77.

52. Confucius, cité dans F. Jullien, La Grande image n'a pas de forme (ou du non-objet par la peinture), Paris, Seuil, coll. «L'Ordre philosophique», 2003, p. 74. 
de manière plus proche encore, chez le peintre Wang Wei : «[...] une eau se perçoit par le vent qui la parcourt ${ }^{53} »$. Mais le vent, tout comme le livre, est associé par Deleuze, en référence à Mallarmé, au pli qui caractérise selon lui le Baroque : «Le pli est inséparable du vent. Ventilé par l'éventail, le pli n'est pas celui de la matière à travers lequel on voit, mais celui de l'âme dans laquelle on lit, "plis jaunes de la pensée", le livre ou la monade aux multiples

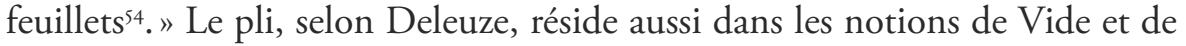
Plein de la pensée extrême-orientale. Pour revenir au tableau d'Antonello da Messina, de récentes études ont démontré que le livre ouvert l'est à la page du «Magnificat». Ce même «Magnificat» qui, dans la composition de Monteverdi, conclura le film, alors que Blanche a déjà pris la posture exacte de la Vierge, en adoptant le même geste des mains, tandis que son châle bleu recouvre ses épaules. «Dans toute conception spiritualiste», écrit Green, «le monde a deux aspects, l'un qui est facilement appréhensible par les sens, et l'autre qui reste caché. Mais le vent qui, invisible, traverse une plaine, n'est pas moins présent, ni moins réel, que lorsque, en faisant plier un arbre, il devient saisissable par la vue», qui ajoute : "La mystique et le cinématographe ont comme vocation la connaissance de ce qui est caché dans le visibless."

Monteverdi est un troisième terme artistique en présence, puisque le film s'ouvre par le "Pulchra es» (repris en son centre, lorsque Virgile met un CD), et se clôt par le mouvement concertato du "Magnificat» ("Magnificat anima mea dominum», "Mon âme exalte le Seigneur»), deux passages des Vêpres de la Vierge (I6Io). Le motet "Pulchra es», pour deux voix féminines, est le second motet des Vêpres, dont le texte est issu du Cantique des cantiques, lieu de fusion entre amour sacré et amour profane. Les vers entendus commencent par «Pulchra es, amica mea» ("Tu es belle, mon amie») et s'achèvent par : "Averte oculos tuos a me, / qui a ipsi me avolare fecerunt» ("Détourne de moi tes yeux / Car ils m'ont vaincu ». Le chef d'orchestre Marco Mencoboni revient sur la traduction, en se référant au texte hébreu originaire : «la phrase correspondant à notre passage est "sheem hirhivuni...", c'est-à-dire "car ceux-ci me troublent", où le mot qui nous intéresse est "hirhivuni", qui signifie précisément troubler, bouleverser ${ }^{56}$ [...]». Mencoboni, s'interroge plus loin : «Il serait utile de savoir quelle était la lecture des textes sacrés à l'époque de Monteverdi à Mantoue et à Venise, quelles

53. Wang Wei, cité dans F. Cheng, Souffle-Esprit. Textes théoriques chinois sur l'art pictural, Paris, Seuil, coll. «Points Essais», 2006, p. II4.

54. G. Deleuze, Le Pli. Leibniz et le baroque, Paris, Minuit, coll. "Critique», 1988, p. 43.

55. E. Green, Poétique du cinématographe, ouvr. cité, p. 21.

56. M. Mencoboni, "Face aux Vêpres de Claudio Monteverdi», livret du CD Cantar lontano [en ligne], E Lucevan le stelle records, p.6. Disponible sur < www.elucevanlestelle.com/ allrecords/monteverdi_vespers_graphics/booklet_FRA_EN_pdf> (consulté le 25/06/20I4). 
étaient les réflexions que faisaient les rabbins, quelle influence a subi notre compositeur (qui était également alchimiste) de la part de ses collègues musiciens juifs et s'il connaissait l'hébreu ${ }^{57}[\ldots]$... Le dialogue amoureux que constitue le Cantique des cantiques s'inscrit en filigrane de la présente relation entre Blanche et Virgile. Pour son film, Green a retenu la version des Vêpres dirigées par Jordi Savall, avec les solistes de la Capella reial de Catalunya et du Coro del Centro musica antica di Padova, enregistrée dans le lieu de création probable de l'œuvre, la basilique palatine de Sainte Barbara : "forme de pèlerinage aux origines", comme l'écrit Savall, qui évoque encore "ces moments miraculeux" où était atteinte une "dimension mystique et presque surnaturelle $e^{58}$. Le choix de Green intègre certainement cette recherche d'une coïncidence entre espace et musique, dans la constitution d'un espace sonore dont la magie pourrait se déplacer et envahir les chambres de Virgile et de Blanche. La musique contribue à relier les espaces disjoints, au moment de la reprise du «Pulchra es" sur la chaîne stéréo. Monteverdi renvoie, à son tour, à Bresson qui utilisa le "Magnificat» dans Mouchette et "Ego dormio " (extrait également du Cantique des cantiques) dans Le Diable probablement. Green avait déjà cité lui-même à plusieurs reprises le «Lamento della Ninfa» dans son film Le Pont des arts (2004).

Une quatrième œuvre fait résurgence dans Correspondances, d'ordre littéraire. Présence visuelle, répétée, d'un livre de la collection «GF» des éditions Flammarion, dont le titre est clairement lisible : La Princesse de Clèves. Livre que lit parfois Blanche, ou que l'on voit posé sur le fauteuil, parfois aux côtés du chat. Quelques similitudes se font jour. L'évocation d'un bal tout d'abord, où se sont rencontrés Blanche et Virgile (au nom surprenant dans le film, puisque nommé «le bal des Martiens-rockers»). La correspondance épistolaire elle-même, puisqu'une lettre est centrale dans le roman de Madame de Lafayette (tant et si bien que Oliveira intitula La Lettre la libre interprétation qu'il en fit en 1999). Bresson avait, en 1953, lui-même écrit un scénario d'après La Princesse de Clèves, qu'il ne put réaliser. Il s'agit bien, ici encore, d'une histoire d'amour impliquant trois personnages dont l'un meurt. Quant à la mère, dans le film de Green, il est possible de penser que son silence perpétuel et sa présence presque fantomatique la situent du côté de la mort et que son départ, comme celui d'Eustache, libère Blanche de l'emprise des disparus.

Du côté des textes, quelques titres se laissent encore lire sur la tranche des livres d'un rayonnage de la chambre de Virgile, San Carlos de Jules Verne, les Pensées de Pascal, et L'Étoile mystérieuse d'Hergé. Mystères de l'imaginaire et de l'esprit s'entremêlent dans un mélange des genres qui correspond bien à cet

57. Ibid., p. Io.

58. J. Savall, sur <http://www.alia-vox.com/cataleg.php?id=68>. 
entrecroisement permanent entre ironie et jeu d'une part, gravité et profondeur d'autre part, qui caractérise les films de Green.

\section{L'art de la conversion}

Ce qui a lieu dans le film est bien une conversion amoureuse, née du tissage de toutes les formes de correspondances. Correspondance épistolaire, correspondances entre les objets, les matières, la matière et l'esprit, la parole et l'image, les œuvres (picturale, littéraire, musicale, filmique) constituent en fait un véritable travail poétique autour de la vision. Le film nait du noir de l'image, dans la musique de Monteverdi, la déclaration amoureuse du "Pulchra es», avant que n'apparaisse la flamme de la bougie qui vient éclairer faiblement l'espace. Et tout le dialogue - finalement virtuel, dans l'échange des voix off comme médium de la révélation de la pensée - tourne autour de cela : convaincre Blanche, prise dans le dogme matérialiste occidental qui prédomine depuis le $\mathrm{XVIII}^{\mathrm{e}}$ siècle, et que Green nomme "atticiste», de l'évidence mystique de leur amour. La flamme de la bougie est le début de la mise en lumière de la présence réelle des sentiments entre Virgile, qui a été marqué par la vision de Blanche, et Blanche qui ne se souvient pas de l'apparence de Virgile. Virgile commence par affirmer à Blanche : "Je pense tellement à vous, que vous êtes présente auprès de moi, comme un fantôme qu'on voit mais qui est sans corps" et, lorsqu'il incite Blanche à se remémorer son visage, la jeune femme réplique : "Désolée je ne vois rien", puis "Je ne vois toujours rien", alors qu' un moment de noir se fait en accord avec sa sentence - qui sera, encore une fois, trahi par le tremblement de la flamme de la bougie. Que Virgile annonce que sa seule passion, avant de connaître Blanche, était le cinéma, va dans le sens de cette quête d'une révélation par l'image. "Nous nous sommes vus et tout a été exprimé», dit Virgile, déclarant qu'il voit dans les yeux de Blanche «son reflet», et le "chemin» de la jeune femme. Il s'avère que la présence fantomatique, mais réelle, comme "un corps vivant", d'Eustache empêche Blanche de voir. "C'était un garçon qui me regardait et que je n'ai jamais vu», la réplique de Blanche montre bien qu'elle fut aveugle à l'amour d'Eustache, tout comme elle l'est encore à celui de Virgile. Mais la conversion se fait peu à peu ressentir lorsque Blanche déclare : "Vous m'avez dit que cet amour existe en vous, Virgile. Vous l'avez fait exister en moi, Blanche. Il est donc réel.» Il s'agit dès lors d'exposer cet amour "à la lumière du jour». La conversion, puisqu' elle est à la fois amoureuse et mystique, passe alors par l'image de l'Annonciation. Croyance dans l'image quaffirme Virgile : "Je crois en beaucoup de choses. Je vois partout des images qui ont un sens. " Il s'agit donc d'aider Eustache, "présence qui a 
vu le vide creux» à trouver «le vide plein » dans «la lumière de la parole». Ces réflexions successives atteignent au cour de la poésie qui consiste, comme l'avait si bien dit Paul Éluard, à donner à voir. "Je crois que toutes les images qu'on voit forment une image plus grande, qui restera toujours invisible. Je ne peux imaginer à quoi elle ressemble, et je ne peux imaginer qu'elle n'existe pas ", dit Virgile, et lorsque Blanche est enfin prête à se libérer d'Eustache - le voyant pour la première fois, au-delà de sa mort, venu reprendre son bonnet bleu -, Virgile la guide en lui disant : "Ne fermez pas les yeux, Blanche, ne fermez pas les yeux. Vous verrez le signe de notre amour.» Blanche peut alors conclure: "J'ai vu la parole que vous m'avez annoncée», et enfin recouvrer une partie de sa mémoire visuelle lorsqu'elle déclare : «c'est vrai, je me souviens maintenant de votre bonnet", et qu'elle en énonce la couleur : "rouge vif» - un large sourire éclairant alors le visage de Virgile. La rencontre au présent pourra se faire, scellée par cet ultime dialogue : "Je pars maintenant pour le square. Venez-vous?» (Virgile), «Oui, je viens» (Blanche).

Les amants vont se rejoindre, mais en dehors de l'espace filmique, horschamp, hors-temps, dans les marges blanches du poème, puisque c'est en leur absence que la présence réelle va encore s'affirmer. À la fin du film, Blanche décide de quitter sa chambre (sa prison, sa cellule de couvent), et c'est à ce moment seulement qu'elle laisse la porte grande ouverte (sa mère, lors de sa sortie ultime, précédant sa disparition symbolique, l'avait elle-même laissée entrouverte comme une invitation au voyage). Dans l'espace vide, la caméra cadre enfin frontalement la fenêtre que la jeune fille avait précédemment ouverte, puis dans un mouvement avant dépasse et efface bientôt le cadre de bois, au profit du cadre de l'image. Sont alors vus les toits de la ville, un pigeon qui s'envole, tandis qu'un panoramique ascendant révèle un coin de ciel au-dessus des cheminées : espace vide laissant se révéler une présence réelle, filmiquement poétique, jusque dans l'obscurcissement final de l'image où renaît la musique du "Magnificat» de Monteverdi venue confirmer que la révélation a bien eu lieu. 